\title{
ON SEMI-ARTINIAN MODULES AND INJECTIVITY CONDITIONS
}

\author{
by J. CLARK AND P. F. SMITH \\ (Received 23rd May 1994)
}

It is well known that a module $M$ has finite length if and only if it is semi-artinian and Noetherian or, equivalently, semi-noetherian and artinian. Our main result shows that finite length is often achieved by just assuming that $M$ is semi-artinian, semi-noetherian and has finitely generated socle.

1991 Mathematics subject classification: 16P20, 16D50, 16L60.

\section{Introduction}

Throughout $R$ is a ring with identity and all modules are unital right $R$-modules. The category of all such modules is denoted by mod-R. A module $M$ is called semi-artinian (or a Loewy module) if every non-zero homomorphic image of $M$ contains a simple submodule or, equivalently, if every non-zero homomorphic image of $M$ has essential socle. Dually, a module $M$ is semi-noetherian if every non-zero submodule contains a maximal submodule. It is well known (see, for example, $[1, \S 11]$ ) that a module $M$ has finite length if and only if it is semi-artinian and Noetherian or, equivalently, it is seminoetherian and Artinian. The main result of this note shows that we can often get finite length by just assuming that $M$ is semi-artinian, semi-noetherian and has finitely generated socle. As a consequence of this we can obtain a recent characterization of quasi-Frobenius rings in [9].

The ring $R$ is called right semi-artinian (respectively right semi-noetherian) if the right $\boldsymbol{R}$-module $\boldsymbol{R}$ is semi-artinian (semi-noetherian). It is well known (see, for example, [10, Proposition 22.32]) that if $R$ is right semi-artinian then each non-zero $M$ in mod- $R$ is also semi-artinian. Chapter 22 of [10] contains further information on semi-artinian rings and rings for which every module is semi-noetherian, therein called socular and $B$ rings respectively. The relationship between these two classes of rings is considered in [24]. In particular [24, Théorème 3.1 ] shows that any commutative semi-artinian ring is semi-noetherian.

For any module $M, \mathrm{E}(M)$ will denote its injective hull. The socle of $M$ will be denoted by $\operatorname{Soc}(M)$. The second socle $\operatorname{Soc}_{2}(M)$ is the submodule of $M$ containing $\operatorname{Soc}(M)$ such that $\operatorname{Soc}_{2}(M) / \operatorname{Soc}(M)=\operatorname{Soc}(M / \operatorname{Soc}(M))$. 


\section{Results}

Our first lemma features in [6, Remarks (2), (3)] and in [4, Proposition 4.4 and Corollary 4.5]. (An analogue also appears in [3, Lemmas 2.1-2.3] with the descending chain condition on essential right ideals instead of the semi-artinian condition.)

Lemma 1. (1) Let $\left\{R_{\lambda}: \lambda \in \Lambda\right\}$ be a (non-empty) collection of right semi-artinian rings and let $R=\prod_{\Lambda} R_{\lambda}$. Then $R$ is right semi-artinian if and only if $\Lambda$ is finite.

(2) Let $R$ be a right semi-artinian right or left self-injective von Neumann regular ring. Then $R$ is semiprime Artinian.

In the seminal paper [5], Bass shows in his Theorem $P$ that every left perfect ring is right semi-artinian (see also [1, Theorem 28.4]). We now note that the converse is true in the presence of self-injectivity.

Proposition 2. Let $R$ be a right or left self-injective ring. Then $R$ is left perfect if and only if $R$ is right semi-artinian.

Proof. Suppose that $R$ is right semi-artinian and let $J$ denote the Jacobson radical of $R$. Then $J$ is left $T$-nilpotent [1, Remark 28.5] and $R / J$ is right or left self-injective von Neumann regular [10, Theorem 19.27]. By Lemma 1(2) and [1, Theorem 28.4], $R$ is left perfect.

Corollary 3. Let $R$ be a right or left self-injective ring. Suppose that $R$ is right semi-artinian. Then $R$ is left semi-noetherian.

Proof. By [1, Theorem 28.4].

The converse to Corollary 3 is not true in general. To see this, let $K$ be any field and $R=\prod_{n=1}^{\infty} K_{n}$, where $K_{n}=K$ for each $n \geqq 1$. Then $R$ is a commutative self-injective von Neumann regular ring, so that $R$ is semi-noetherian (being a $V$-ring), by [20, Theorem 2.1]. However, $R$ is not semi-artinian by Lemma $1(1)$.

A well known open question in ring theory asks whether a right and left perfect right self-injective ring $R$ must be quasi-Frobenius. In [9] this was answered affirmatively under the additional assumption that the second right $\operatorname{socle} \operatorname{Soc}_{2}(R)$ is finitely generated. Our next corollary extends this result.

Corollary 4. Let $R$ be a right and left semi-artinian right self-injective ring such that $\operatorname{Soc}_{2}\left(R_{R}\right)$ is a finitely generated right ideal. Then $R$ is a quasi-Frobenius ring.

Proof. By Proposition 2 and [9, Theorem].

We aim to generalise Corollary 4. First we prove the main result of this note. Recall that a module is finitely cogenerated if its socle is essential and finitely generated. 
Theorem 5. Let $R$ be a ring satisfying the property:

(*) $\quad \operatorname{Soc}_{2}(\mathrm{E}(U))$ is finitely generated for each simple right $R$-module $U$.

Then a right $R$-module $M$ has finite length if and only if $M$ is semi-artinian, seminoetherian and $\operatorname{Soc}(M)$ is finitely generated.

Proof. The necessity is clear. Conversely, suppose that $M$ is semi-artinian, seminoetherian and $\operatorname{Soc}(M)$ is finitely generated. Suppose that $M$ is not Artinian. By Zorn's Lemma there exists a submodule $P$ of $M$ minimal in the collection of submodules $L$ of $M$ such that $M / L$ is not finitely cogenerated (see [1, Proposition 10.10]).

Because $M$ is semi-artinian, $M / P$ has essential socle. Thus $\operatorname{Soc}(M / P)$ is not finitely generated. Note that $P \neq 0$ and, because $M$ is semi-noetherian, $P$ contains a maximal submodule $Q$. By the choice of $P, M / Q$ is finitely cogenerated and hence $\operatorname{Soc}(M / Q)$ is finitely generated. Thus, without loss of generality we can suppose that $Q=0$. In this case, $\operatorname{Soc}_{2}(M)$ is not finitely generated, because $\operatorname{Soc}(M / P)$ is not finitely generated.

Let $S_{1}=\operatorname{Soc}(M)$. There exists a positive integer $n$ and simple submodules $U_{i}$ for $1 \leqq i \leqq n$ of $M$ such that $S_{1}=U_{1} \oplus \cdots \oplus U_{n}$. Then, without loss of generality, $M$ is a submodule of $X=\mathrm{E}\left(U_{1}\right) \oplus \cdots \oplus \mathrm{E}\left(U_{n}\right)$ and so $M / S_{1}$ is a submodule of $X / S_{1} \simeq$ $\left[\mathrm{E}\left(U_{1}\right) / U_{1}\right] \oplus \cdots \oplus\left[\mathrm{E}\left(U_{n}\right) / U_{n}\right]$. By property $(*), \operatorname{Soc}_{2}(X)$ is finitely generated and hence $\operatorname{Soc}_{2}(M) / S_{1}$ is also finitely generated. It follows that $\operatorname{Soc}_{2}(M)$ is finitely generated, a contradiction. Thus $M$ is Artinian and so has finite length.

Our theorem has the following corollaries.

Corollary 6. Let $R$ be a ring for which there exists an injective cogenerator $X$ for mod- $R$ such that $\operatorname{Soc}_{2}(X)$ is finitely generated. Then a right $R$-module $M$ has finite length if and only if $M$ is semi-artinian, semi-noetherian and $\operatorname{Soc}(M)$ is finitely generated.

Proof. If $X$ is an injective cogenerator for mod-R such that the socle of $X / \operatorname{Soc}(X)$ is finitely generated then $\operatorname{Soc}_{2}(\mathrm{E}(U))$ is finitely generated for each simple right $R$-module $U$.

Corollary 7. Let $R$ be a ring and $M$ be an injective cogenerator for mod-R. Then $M$ has finite length if and only if $M$ is semi-artinian, semi-noetherian and $\operatorname{Soc}_{2}(M)$ is finitely generated.

Proof. The necessity is clear. Conversely, suppose that $M$ is semi-artinian, seminoetherian and $\operatorname{Soc}_{2}(M)$ is finitely generated. Clearly $M / \operatorname{Soc}(M)$ has finite Goldie dimension $n$ (say). Suppose that $\operatorname{Soc}(M)$ is not finitely generated. Then

$$
\operatorname{Soc}(M)=S_{1} \oplus \cdots \oplus S_{n+1}
$$

for some non-finitely generated submodules $S_{i}(1 \leqq i \leqq n+1)$. Thus 
and hence

$$
M=\mathrm{E}\left(S_{1}\right) \oplus \cdots \oplus \mathrm{E}\left(S_{n+1}\right)
$$

$$
M / \operatorname{Soc}(M) \simeq \bigoplus_{i=1}^{n+1} \mathrm{E}\left(S_{i}\right) / S_{i}
$$

It follows that there exists $1 \leqq j \leqq n+1$ such that $\mathrm{E}\left(S_{j}\right)=S_{j} \leqq \operatorname{Soc}_{2}(M)$, which is finitely generated. Being a direct summand of $\operatorname{Soc}_{2}(M), S_{j}$ is finitely generated, a contradiction. It follows that $\operatorname{Soc}(M)$ is finitely generated. By the theorem, $M$ has finite length.

We note that Corollary 4 above is now a consequence of Corollary 7 (taking $M=R$ ), Corollary 3 and the well-known fact that any right self-injective right Artinian ring is quasi-Frobenius.

\section{Remarks and examples}

(1). Let $R=\mathbb{Z}$, the ring of integers. Then

(i) the $R$-module $R$ is Noetherian (whence semi-noetherian) with zero socle but is not Artinian,

(ii) for any prime $p$, the Prüfer $p$-group is an Artinian (whence semi-artinian) $R$ module with simple socle but is not Noetherian, and

(iii) any non-finitely generated semisimple $R$-module is semi-artinian and seminoetherian but does not have finite length.

Thus is is not clear how the theorem can be improved.

(2). Following J. P. Jans [16], a ring $R$ is called right co-Noetherian if every factor module of every finite cogenerated right $R$-module is again finitely cogenerated or, equivalently, every finitely cogenerated right $R$-module is Artinian. As a consequence of results of P. Vámos [26], $R$ is right co-Noetherian if and only if each simple right $R$ module has an Artinian injective hull. Thus any right co-Noetherian ring $R$ satisfies property $(*)$ of Theorem 5 . In what follows we give some indication of the ubiquity of co-Noetherian rings.

Firstly, Vámos [loc. cit] has shown that a commutative ring is co-Noetherian if and only if each localization $R_{M}$ is Noetherian for all maximal ideals $M$ of $R$. Also, trivially, every right $\mathrm{V}$-ring is right co-Noetherian.

Theorem 2 of Jategaonkar [17] states that the injective hull of a simple module over a Noetherian P. I. ring is an Artinian module. Consequently any Noetherian P. I. ring is co-Noetherian. On the other hand, Example 7.14 of Chatters and Hajarnavis [7] shows that there are Artinian rings $R$ which do not satisfy property (*) of Theorem 5. More specifically, let $D$ be a division ring with a subdivision ring $K$ such that $D$ is finitedimensional as a left vector space over $K$ but not as a right vector space over $K$. Let

$$
R=\left[\begin{array}{cc}
K & D \\
0 & D
\end{array}\right]
$$


Then $R$ is left and right Artinian. Moreover $M=D e_{11}+D e_{12}$ is a right $R$-module which is an essential extension of the simple right $R$-module $N=D e_{12}$ and the $R$-submodules of $M / N$ correspond to the right $K$-subspaces of $D$ so that $M / N$ is not Artinian. It follows that the socle of $\mathrm{E}(N) / N$ is not finitely generated.

Gupta and Varadarajan have shown [15, Proposition 2.14] that a ring $R$ is right coNoetherian if and only if there is a cogenerator for mod- $R$ which is a direct sum of Artinian modules. They also consider when the endomorphism ring of a finitely generated quasi-projective module is left co-Noetherian. Some of their arguments have been generalised by Garcia Hernández and Gómez Pardo [14].

From Theorem 4 of S. Singh [25] it follows that any hereditary Noetherian prime ring is also co-Noetherian.

Theorem A of I. Musson [22] implies that if $R$ is the group ring $S[G]$ where $G$ is a polycyclic-by-finite group and the coefficient ring $S$ is either $\mathbb{Z}$ or an absolute field then $R$ is co-Noetherian. Also the Main Theorem of [23] shows that if $K$ is a non-absolute field and $G$ is a polycyclic-by-finite group then $K[G]$ is co-Noetherian if and only if $G$ is abelian-by-finite. In fact, Theorem 3.1 of [23] provides another example of a Noetherian ring which does not satisfy Theorem 5's property (*) by proving that if $K$ is a nonabsolute field and $G$ is a nilpotent-by-finite group which is not abelian-by-finite then there is a simple $K[G]$-module $V$ such that $\operatorname{Soc}(\mathrm{E}(V) / V)$ is not finitely generated.

(3). Following Vámos [27], a ring $R$ is defined to be (right) classical if $\mathrm{E}(V)$ is linearly compact for each simple right $R$-module $V$. An account of linearly compact modules can be found in the recent monograph by Xue [28]. In particular, Proposition 3.4 there shows that linearly compact modules have finite Goldie dimension. Consequently, classical rings satisfy property (*) of Theorem 5. Moreover, by Lemma 3.1 of [28], every Artinian module is linearly compact and so right co-Noetherian rings are classical. In view of this, we now give a brief discussion of classical rings.

From Vámos [loc. cit] it follows, using results of Matlis [18] and Müller [21] respectively, that almost maximal valuation rings are classical and any commutative ring with a Morita duality is also classical. Indeed, Pham Ngọc Ánh [2] has recently characterized commutative classical rings as being those rings $R$ for which the completion of the localization $R_{M}$ of $R$ at each maximal ideal $M$ has a Morita duality.

Vámos [loc. cit] defines an $R$-module $M$ to be subdirectly irreducible if $\mathrm{E}(M) \simeq \mathrm{E}(V)$ for some simple $R$-module $V$ or, equivalently, if $M$ has a simple essential socle. He also defines a commutative ring $R$ to be a $S I S I$ ring if, for each ideal $I$ or $R$, the factor ring $R / I$ is self-injective if the $R$-module $R / I$ is subdirectly irreducible. He proves [27, Proposition 3.2] that a commutative classical ring is SISI but gives the following example $R$ to show that the converse is false. (A similar example appears in Section 1.11 of C. Menini and A. Orsatti [19].)

Let $F$ be a field, let $P=F\left[x_{1}, x_{2}, x_{3}, \ldots\right]$ be the polynomial ring over $F$ in a countable number of indeterminates, let $I$ be the ideal of $P$ generated by the set of products $\left\{x_{i} x_{j}\right.$ : $i \geqq 1, j \geqq 1\}$, and let $R=P / I$. Then $R$ is a local ring and its maximal ideal $M$ is a countable direct sum of copies of $R / M \simeq F$. Moreover $\bar{E}=\mathrm{E}(R / M) /(R / M)$ is an infinite direct sum of copies of $R / M$ (see [27] for details). Thus $R$ is not classical and does not satisfy Theorem 5's property (*). 
For further information about SISI rings see [11], [12].

(4). We now give an example of a commutative ring $R$ which satisfies property (*) but is not classical. The ring in question featured in [8] (for other purposes) and we follow its presentation given there.

Let $A$ be a discrete valuation ring with maximal ideal $I=A t$ and quotient field $K$. Moreover assume that $A$ is countable, so that $A$ is not complete in the $I$-adic topology. Let $M$ denote the $A$-module $K / A$ and, for each positive integer $n$, let $M_{n}$ denote the $A$-submodule $A t^{-n} / A$ of $M$ where

$$
A t^{-n}=\left\{a t^{-n}: a \in A\right\}=\left\{u t^{k}: u \text { is a unit in } A, k \in \mathbb{Z}, k \geqq-n\right\}
$$

Then $M$ is the direct union $\bigcup_{n=1}^{\infty} M_{n}$ and any proper nonzero. $A$-submodule of $M$ is $M_{n}$ for some $n \geqq 1$.

Now let $R=A \oplus M$ be the trivial extension of the ring $A$ by its module $M$. Then the nonzero ideals of the ring $R$ properly contained in $M$ are precisely the $A$-submodules $M_{n}$ for each $n$ while the other nonzero ideals of $R$ each contain $M$ and, apart from $M$ and $R$, are of the form $R t^{n}=A t^{n} \oplus M$, with $M=\bigcap_{n=1}^{\infty} R t^{n}$. In fact the ideals of $R$ are linearly ordered, forming the following chain:

$$
0 \subset M_{1} \subset M_{2} \subset \cdots \subset M_{n} \subset \cdots \subset M \subset \cdots \subset R t^{n} \subset \cdots \subset R t \subset R
$$

Thus $M_{1}$ is, up to isomorphism, the unique simple $R$-module and $\mathrm{E}\left(M_{1}\right) \simeq \mathrm{E}(R)$.

Since $M$ is a faithful injective $A$-module, it follows from the discussion on page 22 of [13] that $\mathrm{E}(R) \simeq \operatorname{End}_{A}(M) \oplus M$. Moreover, by arguments in [8], End ${ }_{A}(M) \simeq \hat{A}$, where $\hat{A}$ is the $I$-adic completion of $A$. Since $A$ is countable and $\bigcap_{n=1}^{\infty} I^{n}=0$, we can regard $A$ as properly embedded in $\hat{A}$. It follows that $R$ is not self-injective and has $\hat{A} \oplus M$ as injective hull. Since $(\hat{A} \oplus M) / M_{1} \simeq \hat{A} \oplus M$ as $R$-modules and the latter has $M_{1}$ as a unique minimal submodule, it follows that $\mathrm{E}\left(M_{1}\right) / M_{1}$ has finitely generated socle. Hence $R$ satisfies property (*).

It remains to see that $R$ is not classical. For this, consider the countable set of congruences in $R$ given by

$$
\left\{x \equiv \sum_{k=1}^{n} t^{k}\left(\bmod R t^{n+1}\right): n \in \mathbb{N}\right\}
$$

Then, for any fixed $n$, setting $x=\sum_{k=1}^{n} t^{k}$ gives a simultaneous solution to the first $n$ of these congruences. However there is no simultaneous solution to the complete set of congruences. Hence $R$ is not linearly compact. Thus $\mathrm{E}(R)=\mathrm{E}\left(M_{1}\right)$ is not a linearly compact $R$-module and so, since $M_{1}$ is the only simple $R$-module up to isomorphism, it follows that $R$ is not classical. 
In fact, $R$ is not SISI. To see this, note that the $R$-module $R$ is subdirectly irreducible yet $R$ is not a self-injective ring, and so, with $I$ as the zero ideal, $R$ fails to satisfy the definition of an SISI ring.

(5). From the above remarks we have the following strict implications for any ring $R$ :

$$
R \text { is co-Noetherian } \Rightarrow R \text { is classical } \Rightarrow R \text { has property (*). }
$$

Acknowledgements. This paper was born on a visit by the first author to the University of Glasgow. He thanks the Edinburgh Mathematical Society and the University for their generous financial support and the Department of Mathematics for their kind hospitality.

The authors also thank Claudia Menini for her helpful comments on an earlier version of this paper.

\section{REFERENCES}

1. F. W. Anderson and K. R. Fuller, Rings and categories of modules (Springer-Verlag, Berlin, 1974).

2. Phạ Ngọc Ánh, Morita duality for commutative rings, Comm. Algebra 18 (1990), $1781-1788$.

3. E. P. Armendariz, Rings with DCC on essential left ideals, Comm. Algebra 8 (1980), 299-308.

4. G. BaCCELla, Semi-artinian $V$-rings and semi-artinian von Neumann regular rings, J. Algebra, to appear.

5. H. Bass, Finitistic dimension and a homological generalization of semi-primary rings, Trans. Amer. Math. Soc. 95 (1960), 466-488.

6. V. P. Camillo and K. R. Fuller, On Loewy length of rings, Pacific J. Math. 53 (1974), 347-354.

7. A. W. Chatters and C. R. Hajarnavis, Rings with chain conditions (Pitman, London, 1980).

8. J. ClaRK, On a question of Faith in commutative endomorphism rings, Proc. Amer. Math. Soc. 98 (1986), 196-198.

9. J. Clark and Dinh van Huynh, A note on perfect self-injective rings, Quart. J. Math. Oxford 45 (1994), 13-17.

10. C. Falth, Algebra II: Ring Theory (Springer-Verlag, Berlin, 1976).

11. C. FAITH, Lineary compact injective modules and a theorem of Vámos, Publ. Sec. Math. Univ. Autónoma Barcelona 30 (1986), 127-148.

12. C. FAtTH, Polynomial rings over Jacobson-Hilbert rings, Publ. Sec. Math. Univ. Autònoma Barcelona 33 (1989), 85-97.

13. R. M. Fossum, P. A. Griffith and I. Reiten, Trivial Extensions of Abelian Categories (Lecture Notes in Mathematics 456, Springer-Verlag, Berlin, 1975).

14. J. L. Garcia Hernández and J. L. Gómez Pardo, On endomorphism rings of quasiprojective modules, Math. Z. 196 (1987), 87-108.

15. A. K. Gupta and K. Varadarajan, Modules over endomorphism rings, Comm. Algebra 8 (1980), 1291-1333.

16. J. P. JANs, On co-Noetherian rings, J. London Math. Soc. (2) 1 (1969), 588-590.

17. A. V. Jategaonkar, Certain injectives are Artinian, in Noncommutative ring theory (Intern. Conf., Kent State Univ., Kent, Ohio, 1975) (Lecture Notes in Mathematics 545, Springer-Verlag, Berlin, 1976), 128-139. 
18. E. Matlis, Injective modules over Prüfer rings, Nagoya Math. J. 15 (1959), 57-69.

19. C. Menini and A. Orsatti, Topologically left Artinian rings, J. Algebra 93 (1985), 475-508.

20. G. O. Michler and O. E. Villamayor, On rings whose simple modules are injective, $J$. Algebra 25 (1973), 185-201.

21. B. J. Múller, Linear compactness and Morita duality, J. Algebra 16 (1970), 60-66.

22. I. M. Musson, Injective modules for group rings of polycyclic groups I, Quart. J. Math. Oxford 31 (1980), 429-448.

23. I. M. Musson, Injective modules for group rings of polycyclic groups II, Quart. J. Math. Oxford 31 (1980), 449-466.

24. C. NAstásescu and N. Popescu, Anneaux semi-artiniens, Bull. Soc. Math. France 96 (1968), 357-368.

25. S. SiNGH, Quasi-injective and quasi-projective modules over hereditary Noetherian prime rings, Canad. J. Math. 26 (1974), 1173-1185.

26. P. VÁmos, The dual of the notion of "finitely generated", J. London Math. Soc. 43 (1968), 643-646.

27. P. VÀmos, Classical rings, J. Algebra 34 (1975), 114-129.

28. Xue Weimin, Rings with Morita Duality (Lecture Notes in Mathematics 1523, SpringerVerlag, Berlin, 1992).

Department of Mathematics and Statistics

UNIVERSITY OF OTAGO

P. O. Box 56

DUNEDIN

New Zealand

E-mail: jclark@maths.otago.ac.nz
Department of Mathematics

UNIVERSITY OF GlasGow

UNIVERSITY GaRDENS

GlasGow G12 8QW

SCOTLAND

E-mail: pfs@maths.gla.ac.uk 